\title{
LA CONCILIACIÓN EXTRAJUDICIAL COMO GARANTE DEL CUMPLIMIENTO DE LOS COMPROMISOS EN LOS CONFLICTOS SOCIALES
}

\author{
SASACHAKUYKUNAQ KAMACHIQKUNAQ CHANINCHAKUYNIN \\ EXTRAJUDICIAL NISQAPI SAYARIYNINWAN
}

THE EXTRAJUDICIAL CONCILIATION AS A GUARANTOR OF
COMPLIANCE WITH COMMITMENTS IN SOCIAL CONFLICTS

Ernesto Lechuga Pino(1)

Ch'uyanchakuy: Derechuqa wiñallamuchan kuska cambio tecnologicokunawan, sasachakuykunapas chaninchanapaq, chay raykun paqarimun Métodos Alternativos de Resolución de Conflictos niqa (MARC's); Peru suyunchispi injkay chunka qepa watapiraq paqarimun osqayllaman sasachkuykunata allichanapaq. Chay conciliacion nisqa lloqsimun economicamente derechukunta yupanata. Kay ruwayqa imaynata allichan, sistema judicialman thanirichinanpaq. Peru suyu llaqtakunapi imaymana chaqwakuy kan, rikhurimun pachamamanchismanta orqosqakumanta, chay rakun sayapakunku pachamamanchista allin kananpaq. Chay Chaqwakuykunaqa Peru suyunchisman sayarichin chay empreskunaman, llankakkunaman. Chay Chaqwakuykunaqa manaraq allichankuchu, ayllukunaq manan allinta Peru suyuman qawaspanku, llaqtankupi kirichikusqanku rayku, sipichikuqanku rayku. Chay raykun kay conciliacion extrajudicial nisqa lluqsimun kay chaqwkuykunata allinchananpaq.

Yuyaysapa simikuna:T'irakuy, Allinchay, Pacha Mama, Kamachi

(1) Abogado por la Universidad Nacional de San Antonio Abad del Cusco, magister en Ciencias Penales por la Universidad San Martín de Porres y egresado del doctorado en Ciencia Política y Relaciones Internacionales de la Universidad Ricardo Palma.

Ha ejercido función pública en entidades vinculadas a la administración de justicia, desempeñándose como Viceministro de Derechos Humanos y Acceso a la Justicia, Director General de Defensa Pública y Acceso a la Justicia, Gerente Central de la Escuela del Ministerio Público en la Fiscalía de la Nación, Gerente de Desarrollo de la Oficina de Control de la Magistratura del Poder Judicial, así como Director Académico y Director General de la Academia de la Magistratura.

Ejerce docencia en la Escuela de Post-grado de la Universidad de San Martín de Porres en la asignatura de Jurisdicción y Constitución, en el Pre-grado de la Universidad Tecnológica del Perú en las asignaturas de Derecho Procesal Constitucional, Derechos Humanos y Derecho Constitucional, así como en la Academia de la Magistratura a cargo de la asignatura de Gestión Académica. 
Resumen: Una de las expresiones de desarrollo del Derecho, y las necesidades de justicia, es el surgimiento de los Métodos Alternativos de Resolución de Conflictos (MARC's). La Conciliación Extrajudicial tiene como objetivo la solución amigable de los conflictos en plazos breves y a menores costos. Ella ha logrado solucionar eficientemente múltiples casos, ahorrar esfuerzos y recursos al sistema judicial, ya que su filosofía es extrajudicial. En Perú, los conflictos sociales están asociados frecuentemente a la explotación de recursos naturales en una economía primarioexportadora; por ello la defensa del medio ambiente es uno de los principales temas de la agenda pública. Estos conflictos en muchos casos han frenado ambiciosos proyectos de inversión, perjudicado la generación de puestos de trabajo y de riqueza para el erario nacional. Muchos de estos conflictos no se han solucionado o no han satisfecho las demandas de las comunidades involucradas en el conflicto, habiendo dejado una estela de protestas, actos violentos y numerosas víctimas producto de la violencia desatada a raíz los reclamos sociales. Frente a esta problemática, la conciliación extrajudicial es una alternativa al alcance del Estado y las comunidades en conflicto para la negociación de sus reivindicaciones y acuerdos con la garantía del título de ejecución que jurídicamente tiene el acta de conciliación, lo que garantiza el cumplimiento de los acuerdos que se hayan podido realizar. En este contexto, la conciliación extrajudicial constituye una herramienta de seguridad jurídica que podría ser aplicable a los conflictos sociales para beneficio de la sociedad en su conjunto.

Palabras claves: Conflicto social, Conciliación extrajudicial, Recursos naturales. Ejecutabilidad y Seguridad legal.

Abstract: One of the expressions of law development, and the needs of justice, is the emergence of Alternative Methods of Conflict Resolution. The objective of the Extrajudicial Conciliation is the friendly solution of conflicts in short terms and at lower costs. It has been to solve efficiently multiple cases, save efforts and resources to the judicial system, since its philosophy is extrajudicial. In Peru, social conflicts are frequently associated with the exploitation of natural resources in a primary-exporting economy; for this reason, the defense of the environment is one of the main topics on the public agenda. These conflicts have, in many cases, slowed down ambitious investment projects, damaging the generation of jobs and wealth for the national treasury. Many of these conflicts have not been resolved or have not met the demands of the communities involved in the conflict, leaving behind a trail of protests, violent acts and numerous victims as a result of the violence unleashed by social demands. Faced with this problem, the extrajudicial conciliation is an alternative for the State, and the communities in conflict, for the negotiation of their claims and agreements with the guarantee of the execution title that legally has the conciliation act, which guarantees the fulfillment of the agreements that could have been made. In this context, out-ofcourt conciliation constitutes a legal security tool that could be applicable to social conflicts for the benefit of society as a whole.

Key words: Social conflict, Extrajudicial conciliation, Natural resources, Enforceability and security. 


\section{Marco teórico}

El estudio de los conflictos sociales y los mecanismos para su solución, desde un punto de vista jurídico, requiere establecer conceptos básicos que contribuyan a entender el eje de esta problemática e identificar el contexto en el cual se plantean las conclusiones y recomendaciones del presente trabajo. La naturaleza multidisciplinaria de los conflictos sociales involucra conocimientos que provienen de diversas disciplinas del conocimiento humano como son la economía, ecología, antropología, negociación, entre otras; que aportan sus contenidos a la comprensión de este aspecto social.

\subsection{Definiciones}

\section{a) Conflicto social}

Los conflictos sociales constituyen una manifestación de protesta y reivindicación de comunidades o grupos sociales determinados, frente a situaciones que afectan su normal desenvolvimiento o modo de vida. En sociedades en vías de desarrollo o economías primario exportadoras, como la peruana, por lo general están vinculados al desarrollo de actividades extractivas o de explotación de los recursos naturales donde las poblaciones afectadas expresan su rechazo o determinadas exigencias para su realización.

Desde el gobierno, las esferas oficiales y los inversionistas, el conflicto social es percibido como un factor de retraso que afecta el desarrollo económico, generalmente politizado y cuyas demandas asumen posiciones maximalistas de carácter ambiental $u$ otras reivindicaciones sociales, para oponerse a proyectos de inversión y explotación de los recursos naturales u otras circunstancias basadas en el modelo económico del libre mercado.

La Defensoría del Pueblo del Perú considera que el conflicto social "es un proceso complejo en el que sectores de la sociedad, el Estado o las empresas perciben que sus objetivos, intereses, valores o necesidades son contradictorios. Estamos pues ante demandas de numerosas personas que se sienten amenazadas o perjudicadas por la contaminación de un río, la mala prestación de un servicio público, la afectación a sus derechos laborales u otros motivos, y que se movilizan para buscar explicaciones sobre lo ocurrido y encontrar soluciones" (DEFENSORÍA DEL PUEBLO, 2015).

Desde la academia, Karl-Heinz considera como conflicto social a "las divergencias, las tensiones, las rivalidades, las discrepancias, las disputas y las luchas de diferente intensidad entre distintas unidades sociales: entre (y dentro de) los roles sociales, grupos sociales, organizaciones, sectores sociales, sociedades, Estados y entidades supraestatales" (HILMAN, 2001); por su parte Lewis Coser señala que "El conflicto 
social significa una lucha con respecto a valores y derechos sobre estados, poderes y recursos escasos, lucha en el cual el propósito es neutralizar, dañar o eliminar sus rivales" (LEWIS, 1961).

Aunado a esto, Pratt-Fairchild considera que los conflictos se caracterizan por su objetivo inmediato, lo que se manifiesta en: i) el daño que puede causarse a uno o más de los individuos o grupos opuestos, o en los perjuicios que pueden producirse a su propiedad, valores culturales o a cualquier cosa de su apego y afecto, en una dinámica de ataque y defensa; o bien en ii) las actividades de una persona o grupo, que de modo no deliberado, impiden el funcionamiento o dañan la estructura de las instituciones o las empresas (PRATT FAIRCHIL, 1974).

\section{b) Conciliación extrajudicial}

Los mecanismos oficiales de administración de justicia generalmente comprenden la realización de trámites engorrosos, de difícil comprensión para el ciudadano, elevados costos, excesivos formalismos y ritualismos que lo distancian de la población; circunstancias que han favorecido la aparición de los mecanismos alternativos de solución de conflictos como la conciliación extrajudicial, la mediación, la transacción, el arbitraje, la negociación entre otras formas para solucionar las controversias con legitimidad, fuerza de ley y efectividad.

Los mecanismos alternativos de solución de conflictos se inscriben en lo que la doctrina ha venido a llamar como la "cultura de paz", su objetivo es la búsqueda de fórmulas de consenso para la restitución del orden social quebrantado, en contraposición de la litigiosidad donde el conflicto se resuelve unilateralmente mediante una sentencia de cumplimiento obligatorio.

El sistema legal peruano ha elegido como mecanismo alternativo para la solución de conflictos, con validez plena, fuerza legal y naturaleza prejudicial, a la conciliación extrajudicial. La Ley $N^{\circ} 26872$, Ley de Conciliación Extrajudicial, en su artículo $5^{\circ}$ señala que es una institución que se constituye como un mecanismo alternativo para la solución de conflictos, por el cual las partes acuden ante un Centro de Conciliación o al Juzgado de Paz Letrado a fin que se les asista en la búsqueda de una solución consensual al conflicto (CONGRESO DE LA REPÚBLICA). Complementariamente, Rafael Medina Rospigliosi la define como "un medio de solución de conflictos, por el cual un tercero neutral e imparcial denominado Conciliador Extrajudicial asiste a las partes a encontrar su propia solución a sus conflictos que es más humana, saludable, justa, durable, mutuamente satisfactoria y con el mismo valor de sentencia inapelable, es una poderosa herramienta de tercera generación para solucionar conflictos" (MEDINA ROSPIGLIOSI, s.f.). 


\section{c) Recursos naturales}

La Constitución Política de 1993 establece en su artículo 66 que“los recursos naturales, renovables y no renovables, son patrimonio de la Nación. El Estado es soberano en su aprovechamiento...", en esta medida, se otorga la titularidad de dichos recursos a un concepto más amplio que el propio Estado, la Nación que reúne en si a la población, la cultura, el territorio y al propio Estado. En este sentido, la explotación de los recursos naturales constituye un interés nacional, más allá de las aspiraciones locales o justas reivindicaciones de las poblaciones o sectores sociales que ejerzan derechos o residan en la zona de explotación, todo ello, sin negar las facultades de autodeterminación y beneficios que pudiera corresponder a los mismos.

La explotación de los recursos naturales debe realizarse en armonía con las políticas de conservación de la diversidad biológica y el desarrollo sostenible, conforme lo establece la Constitución Política en sus artículos 67 y 68. Las normas de desarrollo constitucional sobre la materia y los tratados internacionales suscritos por el Estado peruano han previsto una serie de mecanismos para armonizar la explotación de los recursos naturales con el interés de las poblaciones y la protección del medioambiente para lo cual se requieren, entre otros, estudios de impacto ambiental, procesos de consulta previa y licencia social; situaciones que han merecido una serie de controversias y polémicas entre la doctrina y la política pública asumida en diversas circunstancias.

Respecto a los recursos naturales, la legislación nacional los define como todos aquellos componentes de la naturaleza susceptibles de ser aprovechados para la satisfacción de las necesidades de los seres humanos y que tienen un valor —actual o potencial- en el mercado (Ley №28611). Asimismo la Organización Mundial del Comercio (WTO - en sus siglas en inglés - World Trade Organization), en el Informe sobre el Comercio Mundial del año 2010, menciona que "Los recursos naturales pueden ser considerados como activos naturales de capital, distintos del capital físico y humano porque no los crea la actividad humana" (COMERCIO, 2010), los distingue así de otros objetos que son susceptibles de comercio pero que no cuentan con las características esenciales e intrínsecas de los recursos naturales como son la agotabilidad y la no intervención del ser humano para su nacimiento y origen, entre otros.

\section{La problemática de los conflictos sociales}

Los conflictos sociales implican indudablemente una problemática que influye tanto en la población como en el Estado ya que tienen consecuencias económicas, políticas y jurídicas. En el Perú, un país reconocido internacionalmente por su megadiversidad y por la exportación de materias primas, los conflictos han ido incrementándose con el transcurso de los años, resaltando los relacionados a 
actividades de extracción y uso de los recursos naturales; así se puede apreciar en el cuadro siguiente:

\section{Canflic1os Sociales}

"Repurle de Conflicilus Sociales"

Enero-Diciembre 2017

T.stado del C.ontlicto Social

\begin{tabular}{cccccc|c}
\hline Categoria & Activos & Litlencia & Retirado & Resuclto & Total \\
\hline$N^{\circ}$ & 119 & 50 & 59 & 28 & 256 \\
\hline \multicolumn{7}{c}{ Clases de Contlicto Social por su alcance y resultados }
\end{tabular}

\begin{tabular}{|c|c|c|c|}
\hline Ambito & Gubicmo Lerial & Gubismo $N$ abiomal & $\begin{array}{c}\text { Demarcacion } \\
\text { Territorial } \\
\end{array}$ \\
\hline $\mathbf{N}^{\circ}$ & $9.8 \%$ & $8.6 \%$ & $5.1 \%$ \\
\hline Resultado & Protcsial & Horidos & Fallecidos \\
\hline $\mathbf{N}^{0}$ & lo09 accioncs & 104 & 06 \\
\hline
\end{tabular}

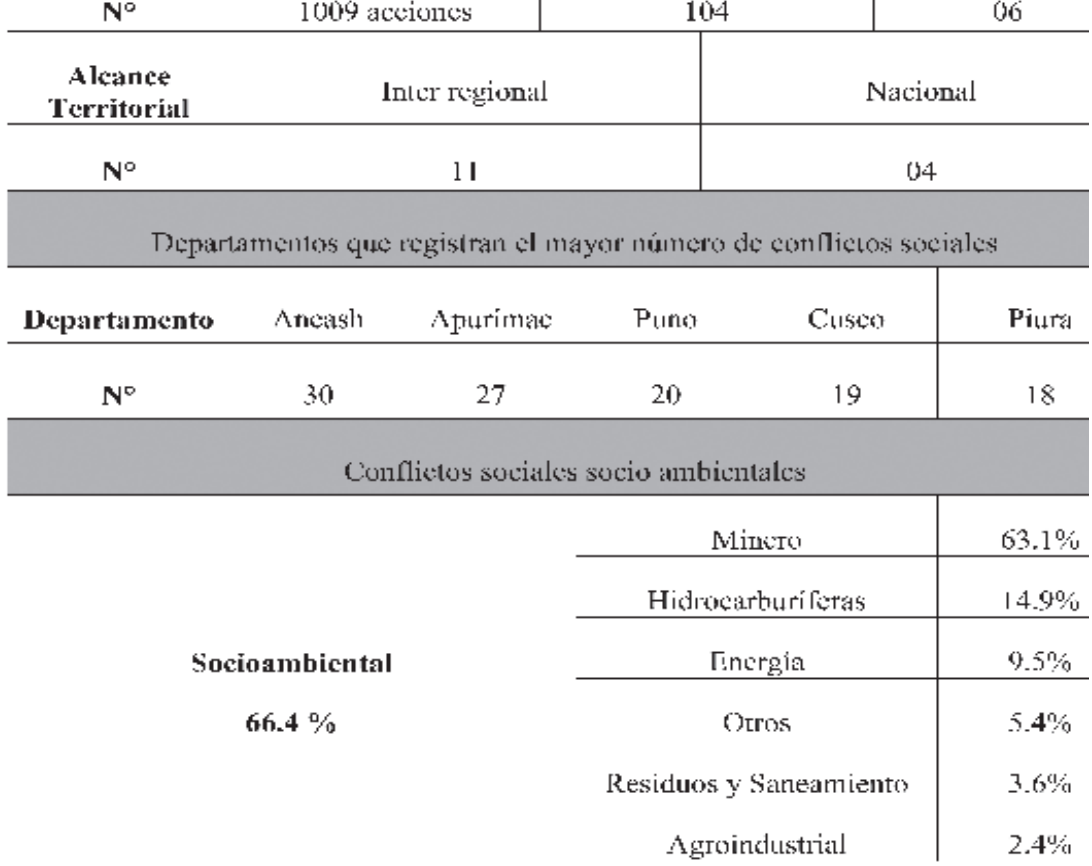

Fuente: Reporte de Conflictos Sociales de la Defensoría del Pueblo del Perú 2017 (Defensoría del Pueblo, 2018a) 
En el último reporte mensual de conflictos sociales, elaborado también por la Defensoría del Pueblo, se señaló que los denominados conflictos socio ambientales continúan predominando representando el 63.6\% (Defensoría del Pueblo, 2018b) del total, aunque en la actualidad varios de ellos responden a la categoría de conflictos latentes, registrándose además alertas tempranas para cuatro casos que pueden devenir en nuevos conflictos.

A lo largo de los años, han surgido diversos conflictos vinculados a la minería, entre los que se pueden mencionar a las localidades y empresas siguientes: Conga y Newmont, en Cajamarca; Tambo Grande y Manhattan en Piura, Quilish y Newmont en Cajamarca, Río Blanco y Rio Blanco Copper en Piura, Tía María y Southern Copper Corporation en Arequipa, así como Santa Ana y Bear Creek en Puno.

Es evidente, que tal como sucede en la actualidad, en el futuro predominaran los conflictos socio ambientales debido a que el Perú posee una variada geografía, biodiversidad siendo un país rico en materias primas y por ende exportador de estas; aunado a ello, la escasez de recursos en diversas partes del mundo genera que tanto las empresas como los Estados busquen en países, como el nuestro, los insumos para la satisfacción de sus necesidades y la realización de sus actividades. Por otro lado, el ciclo de la explotación de los recursos naturales es una actividad que interesa al Estado como fuente generadora de recursos económicos para el erario nacional, se inicia con la identificación de los mercados que demandan el consumo de determinadas materias primas, su extracción, procesamiento e incorporación a los mercados nacionales o internacionales de industrialización o consumo.

Como es de esperarse, la población que reside en las zonas de extracción, tiene la expectativa de i) beneficiarse con las regalías y el canon que genera la explotación de los recursos naturales, y que ii) se atienda o reparen los daños ambientales o la afectación de los ecosistemas que pudiera generar la explotación de un determinado recurso u otra actividad extractiva. Por lo general, estas últimas son las causas detonadoras de la conflictividad social en el Perú y los países latinoamericanos. Esta situación demanda por parte del Estado una actividad proactiva orientada, por un lado, a la prevención de los conflictos socio ambientales $y$, por otro, al cumplimiento de los compromisos que se hayan adoptado, para lo cual, la conciliación extrajudicial se constituye en una alternativa para garantizar la ejecutabilidad de los acuerdos que vinculan al Estado, los inversionistas y las comunidades beligerantes.

\section{Mecanismos usados para la solución de los conflictos sociales}

En el Perú, muchos de los conflictos sociales no se resuelven por la inacción del Estado, y que si bien, no se manifiestan públicamente por el desistimiento de los inversionistas o de la población que fatigada abandona sus reivindicaciones, 
se encuentran en situación de latencia a la espera de una circunstancia detonante que desencadene nuevamente una crisis.

En este contexto, se ha hecho uso de diversas herramientas para intentar solucionar estos conflictos, entre los que se pueden considerar:

a) Instalación de mesas de diálogo: establecidas como un espacio político donde confluyen los protagonistas del conflicto para solucionar sus controversias. Son conformadas generalmente cuando un conflicto se encuentra en su etapa de crisis (PUMA ALMANZA) y constan de tres fases: i) una referida a la apertura de la mesa; ii) otra al dialogo, estructurado en general por el diagnóstico, las soluciones posibles, los responsables y el seguimiento, estas etapas varían de acuerdo a la naturaleza del conflicto; y, iii) culmina con el cierre, donde se hace una síntesis de los consensos. La experiencia, en la utilización de esta herramienta, deja entrever que la complejidad de las negociaciones, especialmente cuando se trata de conflictos socio ambientales, requieren de varias sesiones para alcanzar acuerdos.

b) Instalación de mesas de desarrollo: vinculadas a la estrategia de prevención del conflicto, impulsadas para dar sostenibilidad y monitoreo de los acuerdos arribados, se plantean frente a las limitaciones identificadas en las mesas de diálogo. Su objetivo es estudiar la problemática de un determinado ámbito, discutir soluciones, canalizar inversiones o intervenciones del Estado y aportes de las empresas privadas. Se desarrolla en el marco de una perspectiva de desarrollo integral que aborde los problemas de fondo, trascendiendo a los temas de coyuntura. Se presentan como una alternativa para avanzar en las políticas de diálogo, para fortalecer la presencia del Estado y de sus instituciones, creando un clima de confianza que consolide la gobernabilidad (PUMA ALMANZA).

c) Soluciones normativas: en este caso, el conflicto puede concluir con la promulgación o derogación de una norma específica, sea una ley o cualquier norma vinculada, por ejemplo, al tema de la actividad minera. Tal es el caso del conflicto desatado en Bagua producto de la promulgación de los Decretos Legislativos N 1064 y №1090, que desencadeno la muerte de 23 personas, frente a lo cual el Congreso de la República, a través de la Ley N²9382, publicada el 17 de junio de 2009, derogo estos decretos.

d) Acuerdos: el correcto diálogo entre las partes intervinientes en un conflicto se concretiza a través de la firma de un documento consensual que contendrá los compromisos hechos y la forma en la 
que estos se deben cumplir. Se da entre autoridades, como son los ministros (Poder Ejecutivo), los alcaldes, los gobernadores regionales así como los dirigentes de los grupos y comunidades implicadas.

e) Soluciones represivas: Son todas aquellas respuestas desde el Estado o la autoridad dirigidas a aplacar, por medios violentos, una amenaza o un peligro. Según el Diccionario de la Real Academia de la Lengua Española, es el acto, o conjunto de actos, ordinariamente ejecutados desde el poder, para contener, detener o castigar con violencia actuaciones políticas o sociales. En términos generales se puede sostener que las soluciones represivas, implican el empleo de la fuerza frente a una situación de conflicto donde se causó daño a un individuo o colectividad o se pone en riesgo la seguridad pública y el orden social.

El ordenamiento legal peruano regula el uso de la fuerza por parte de la Policía Nacional del Perú, a través del Decreto Legislativo N¹186, que intentan establecer criterios para que las fuerzas de seguridad, en el marco de sus actuaciones oficiales, realicen un empleo dosificado de la fuerza según las circunstancias para lograr el control de una situación que constituye una amenaza o atenta contra la seguridad, el orden público, la integridad o la vida de las personas; la mencionada norma señala que el uso de la fuerza se realiza de manera progresiva y diferenciada bajo los principios de legalidad, necesidad y proporcionalidad; así como el respeto de los derechos fundamentales.

f) Inacción: generalmente se produce por el abandono de las instituciones del Estado y las autoridades competentes en la implementación de soluciones a reclamos sociales sobre determinada necesidad. En las circunstancias de inacción o latencia, los conflictos van escalando, generando posibles situaciones de crisis.

Cuando es en estas etapas (fase temprana y escalamiento) en las que los conflictos deberían ser identificados y tratados preventivamente a fin de lograr su remediación oportuna. Estas fases son ideales para que el Estado cumpla con su rol de prevención de los conflictos sociales empleando para ello las estrategias de dialogo, consulta previa, estudios de impacto ambiental, así como políticas de control de riesgos frente a situaciones de emergencia.

\section{Costos de los conflictos sociales en el Perú}

Una de las grandes preocupaciones de los conflictos sociales, y específicamente los vinculados a la minería, es el costo económico que han desencadenado en muchos casos con la postergación de varios proyectos mineros de gran envergadura. 
En este sentido, un estudio del Instituto Peruano de Economía (IPE), del año 2015: “...se estima que desde 2011 se han retrasado inversiones mineras por más de 21,500 millones de dólares, el valor anual de la producción asciende a 14,900 millones de dólares a precios del año 2007. En el período 2011-2014 se habría perdido 62,500 millones de dólares de PBI y además a esto se han perdido un promedio de 791,000 empleos por la fase de inversión. Por otro lado, para la fase de producción, desde la maduración de todos los proyectos en 2014, se perderían 1 millón 160 mil empleos anuales" (INSTITUTO PERUANO DE ECONOMÍA, 2015). De acuerdo a este mismo estudio, la economía peruana generaba un producto bruto interno (PBI) cercano a 200,000 millones de dólares y tenía una población económicamente activa (PEA) de algo más de 16 millones de personas; sin embargo, entre el 2011 y el 2014 el Perú habría perdido casi la tercera parte de su PBI anual y la posibilidad de emplear a casi el cinco por ciento de su PEA.

En Perú, la actividad de extracción minera, conforme al estudio del IPE del 2015, representaba más del 50\% de las divisas, el 20\% de la recaudación tributaria y comprende la mayor parte de inversión internacional. Ha aportado recursos para impulsar el crecimiento económico y el desarrollo de las comunidades ubicadas en la zona de influencia de los yacimientos mineros; estos, conforme al artículo 5 de la Ley $N^{\circ} 27506$, Ley de Canon, se distribuyen entre los gobiernos regionales y locales de acuerdo a los índices de distribución que fije el Ministerio de Economía y Finanzas en base a criterios de Población y Necesidades Básicas Insatisfechas:

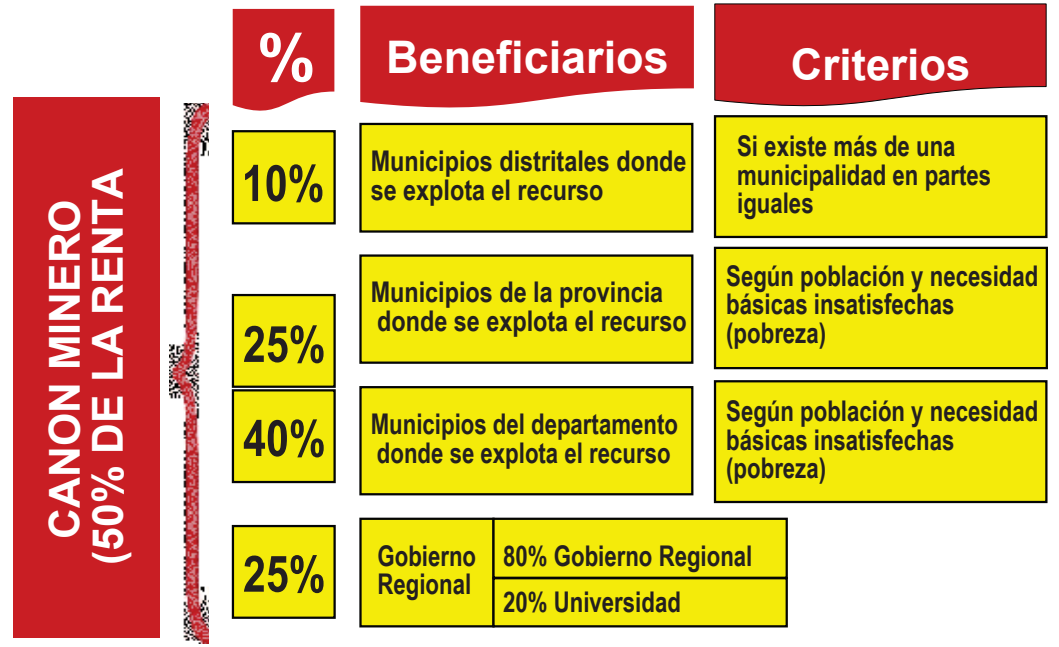

Fuente: Ministerio de Economía y Finanzas

Los sucesivos gobiernos, incluyendo a los locales y regionales, no han logrado canalizar las preocupaciones y reclamos de las comunidades hacia soluciones concertadas, pacíficas y sostenibles. No existe un marco propicio para 
el diálogo y consenso a favor de una convivencia eficiente de la minería con las comunidades; la violencia se ha convertido en el "método de negociación" y como consecuencia se ha incrementado la demanda de soluciones "efectivas" que reclaman la "mano dura" del Estado.

\section{La Conciliación Extrajudicial}

Hace 20 años aproximadamente, a través de la Ley $N^{\circ} 26872$, promulgada el 13 de noviembre de 1997, regreso al Perú la figura jurídica de la conciliación extrajudicial, después de más de 70 años de ausencia en el ordenamiento jurídico, desde la Constitución de 1920 que únicamente preveía la conciliación en conflictos de naturaleza laboral.

Para tratar el tema de la Conciliación Extrajudicial es necesario hacer una breve reflexión sobre el tratamiento de los Métodos Alternativos de Solución de Conflictos (MARC's). Algunos doctrinarios, hacen críticas en relación a la definición de dichas figuras jurídicas, esto básicamente porque en la realidad peruana, cuando alguien tiene un problema jurídico asume que debe acudir al Poder Judicial y no contempla inmediatamente la posibilidad de utilizar los métodos alternativos de solución de conflictos; ello quizá debido a su propia denominación, ya que al asumirse como "alternativos" dan la idea de que existe algo "principal"; así, el Poder Judicial se encumbra como el instrumento por excelencia para la solución de conflictos, los jueces dotados del ius imperium son considerados como los únicos operadores con poder coercitivo, enraizando así en la sociedad peruana una "cultura de litigiosidad".

En este orden de ideas, la práctica de los métodos alternativos como fórmula de solución de los conflictos, es una postura aproximadamente reciente, que se viene consolidando como una alternativa más económica y rápida que la justicia ordinaria.

La razón de optar por la Conciliación Extrajudicial, es el Acta de Conciliación, ya que la ley le otorga la calidad de título ejecutivo, por lo que ante su incumplimiento la parte interesada solamente acudirá a la vía judicial para su ejecución mediante el Proceso Único de Ejecución, que es un trámite más célere; con lo cual se evita la prolongación de la afectación de derechos; convirtiéndose en un acto jurídico, firme y susceptible de ejecución, incluso de manera forzosa.

La sociedad ha evolucionado con el trascurso del tiempo, consecuentemente, la naturaleza de los conflictos sociales también ha cambiado, lo que ha producido la existencia de necesidades y desafíos modernos y más complejos, en todos los aspectos vinculados al desarrollo del ser humano, como lo es el tema socioambiental y la extracción de materias primas. 
El conflicto social dentro de su componente reivindicativo tiene una doble estructura:

1. Por un lado, el macroconflicto, que es el que contiene una carga política y demandas que implican decisiones de alto nivel, actos de gobierno o legislativos que deben ser resueltos en las instancias del gobierno nacional.

2. Y el microconflicto, acompañado de una serie de demandas y compromisos locales, de puntual cumplimiento y fácil realización, estos, en muchos casos, son acordados en mesas de dialogo, negociaciones, acuerdos regionales, entre otros, con el objetivo de diluir el conflicto y alcanzar la paz social. El microconflicto es el más latente al estar en contacto directo con la población, involucra la atención y satisfacción de necesidades inmediatas y de consumo instantáneo, los cuales se pactan mediante documentos sujetos a la voluntad y buena fe de las partes, esta forma tiene efectividad en muchos casos, pero en muchos otros, no se cumplen, trayendo como consecuencia tanto desconfianza como resistencia, desencadenando la"escala de controversias" como un círculo vicioso de reproducción del conflicto.

Es en este escenario que surge la Conciliación Extrajudicial como una herramienta jurídica idónea porque demuestra su real potencialidad y capacidad de restituir la paz social mediante el diálogo y la participación del conciliador (tercero ajeno al proceso que propone alternativas de solución) al lograr que se solucionen los conflictos en armonía de las partes, donde los acuerdos tendrán valor de sentencia y se cumplirán en los términos pactados. Esto, sin duda alguna, conlleva a una serie de responsabilidades que deberán ser verificados por el conciliador antes de incluirse el acta de acuerdos respectiva, esto para garantizar su futura ejecución, por lo que debe existir legalidad y viabilidad en los acuerdos y la declaración expresa de actuar libre y voluntariamente, así como la razonabilidad y proporcionalidad, se suma a esto la capacidad de exigibilidad para el cumplimiento de los acuerdos, la cuantificación económica de los acuerdos adoptados para facilitar su ejecución en caso de incumplimiento, y también son necesarios el cumplimiento de los demás requisitos formales para su validez.

Con el fin de analizar las ventajas de la Conciliación Extrajudicial, se desarrolla el siguiente cuadro comparativo: 


\begin{tabular}{|c|c|}
\hline \multicolumn{2}{|c|}{ Conflicto solucionado: } \\
\hline Vía Judicial Ordinaria & Conciliación Extrajudicial \\
\hline $\begin{array}{l}\text { 1. Duración: largo plazo. } \\
\text { 2. Materias: todas. } \\
\text { 3. Costo: excesivo. } \\
\text { 4. Excesiva carga procesal. } \\
\text { 5. Dependencia de las funciones de } \\
\text { otros órganos del Estado (fiscalía, } \\
\text { procuradores, defensores públicos, } \\
\text { etc.). } \\
\text { 6. Se somete al juez que tenga } \\
\text { jurisdicción. } \\
\text { 7. La sentencia no siempre beneficia a } \\
\text { ambas partes, ya que el que decide } \\
\text { objetivamente es el juez, y puede ser } \\
\text { apelada o incluso ir en casación. }\end{array}$ & $\begin{array}{l}\text { 1. Duración: corto plazo (30 días } \\
\text { calendario). } \\
\text { 2. Materias: las pretensiones } \\
\text { determinadas o determinables que } \\
\text { versen sobre derechos disponibles } \\
\text { de las partes. } \\
\text { 3. Costo: Asequible. } \\
\text { 4. Procesos céleres. } \\
\text { 5. Autónomos, no depende de } \\
\text { investigaciones policiales, fiscales u } \\
\text { otros. } \\
\text { 6. Puede optarse por el Centro de } \\
\text { Conciliación libremente al que se } \\
\text { desee someter el conflicto. } \\
\text { 7. El acuerdo plasmado en el Ac ta de } \\
\text { Conciliación es product o del } \\
\text { consenso entre las partes y ante } \\
\text { incumplimiento se puede recurrir el } \\
\text { Proceso Único de Ejecución. }\end{array}$ \\
\hline
\end{tabular}

Fuente: Elaboración propia.

Debemos indicar que si bien, el propósito de optar por la Conciliación Extrajudicial es no recurrir a un juez para la solución de los conflictos, esto no le quita viabilidad a este mecanismo para recurrir al Poder Judicial solo para los efectos de solicitar el cumplimiento del acta de conciliación estructurada válidamente.

Por su parte el experto mexicano en mediación Jorge Pesqueira Legal, comentado los conflictos sociales mineros en Perú, señala que las autoridades responsables de contener los conflictos sociales deben poseer un sistema que permita indicar el verdadero conflicto, así como hacer viable el diseño de estrategias para evitar las consecuencias propias de un problema social. Señala, 
que el método de preferencia, debe ser el optar por el diálogo a través de la conciliación extrajudicial, permitiéndose de esta manera que las relaciones entre las partes sean más dinámicas, Pesqueira incluso reconoce la importancia de que se tenga un capital humano que facilite la comunicación, de esta manera él plantea que dentro del Ministerio responsable de los temas socio ambientales debe operar un departamento de expertos en diálogo, mediación y conciliación con conocimientos suficientes sobre conflictos socio ambientales y con capacidad para prevenirlos e intervenir en su solución (PESQUEIRA LEAL, 2015).

Esta figura podría ser homologada en el caso peruano ya que deben ser las personas más aptas quienes en primera instancia acudan a la conciliación extrajudicial, y de esta manera busquen acuerdos que sean beneficiosos para las partes intervinientes, como sería, por ejemplo, el caso de la indemnización por la afectación del patrimonio de una colectividad u otros.

\section{Legislación Comparada}

\subsection{Colombia}

La legislación colombiana establece que la conciliación ambiental extrajudicial, es viable en los asuntos susceptibles de transacción y desistimiento, cualquiera que sea su naturaleza: en derecho o en equidad. Por lo que la mayor parte de los asuntos ambientales son conciliables por ser de carácter patrimonial, se trata entonces que con la práctica de la conciliación extrajudicial, sea posible ejecutar de la mejor manera los acuerdos optados por las partes (Ley 23 de 1991 en su artículo 85 como el artículo 65 de la Ley 446 de 1998 y el artículo 19 de la Ley 640 de 2001).

\subsection{Costa Rica}

En este país centroamericano, diversos doctrinarios han abocado el tema respecto a la viabilidad o no de la conciliación extrajudicial, de esta manera han analizado por ejemplo la "disponibilidad de los bienes ambientales". Dentro de este tema, primero es necesario establecer que el término "disponer" puede tener dos aseveraciones, una de ellas será la que se relacione tanto con la conservación como con la protección de los recursos naturales, y otra, en contraste, que se vincula a su uso y aprovechamiento.

En Costa Rica, en el tema de la conciliación, se ha establecido que si será posible hacer uso de los RAC - Resolución Alterna de Conflictos, para Perú MARC's, siempre y cuando estos no afecten derechos de terceros, o se incumplan normas de orden público, e incluso son lo de la postura que de ser el caso de que exista un incumplimiento en un pago por el derecho de explotación de determinado recurso, este conflicto se pueda solucionar mediante la conciliación extrajudicial. 
Respecto a qué tipo de materias tienen o no "disponibilidad" y por ende si tienen la susceptibilidad de ser solucionados por un tercero que no necesariamente sea un juez, se debe considerar que es disponible todo aquello que pueda comercializarse por el hombre, es decir, ser valorado o apreciado y susceptible de reconocimiento jurídico.

Tanto en Costa Rica como en otros países, la excesiva carga procesal que existe en los despachos judiciales y fiscales genera que los conflictos, tanto jurídicos como sociales, se intensifiquen y se alarguen. Surgen así, como una opción, los MARC's, que por sus características tienden a ser una experiencia ágil y rápida. Su práctica es tendente y preferente al dialogo, como consecuencia, casi propia de dicha naturaleza, los conflictos jurídicos ya no se verán como obstáculos y barreras entre las partes, sino como una posibilidad y oportunidad de cambiar, mejorar y afianzar así esa relación ya existente.

El marco jurídico y la doctrina costarricense considera fundamental aplicar en la práctica de la conciliación extrajudicial, para la solución de conflictos socio ambientales, principios del Derecho ambiental:

i) Principio precautorio natura: frente a la duda sea técnica o científica sobre la explotación, uso, cesión u otra figura jurídica de un determinado o determinados recursos naturales y su impacto con el medio ambiente se debe interpretar a favor de estos, asumiendo así una medida de precaución. El fin del Derecho Ambiental no debe ser el sancionar sino prevenir que se cause algún daño tanto a los ecosistemas como a salud de las personas.

ii) Principio de solución a la fuente del problema: se exige que si se produce algún tipo de daño o lesión a los recursos naturales, se corrija dicha afectación en la fuente.

iii) Principio de desarrollo sostenible: referido a la toma de conciencia desde el aspecto del cambio ambiental global, que como ya muchas organizaciones, entre ellas la ONU, han señalado, tiene su origen en el daño a la capa de ozono, la explotación descontrolada de los recursos naturales y la contaminación.

iv) La doctrina adicionalmente ha desarrollado el principio "quien contamina paga", mediante el que se pretende que aquella persona natural o jurídica que realiza cualquier acción lesiva al medio ambiente sea responsable pecuniariamente de las consecuencias.

\section{La Conciliación Extrajudicial como garantía para el cumplimiento de los acuerdos para la solución de los conflictos sociales}

El uso de los MARC's, incluida la conciliación extrajudicial, está limitado a 
aquellas materias y aspectos de carácter patrimonial, y que versen sobre derechos disponibles. Al respecto, pueden existir elementos dentro delos derechos ambientales que no puedan ser solucionados mediante estos mecanismos alternativos, sin embargo, aquellos relacionados a efectos cuantificables, es decir de valoración dineraria, si pueden ser objeto de conciliación entre las partes intervinientes.

En fomento de la protección del medio ambiente y los recursos naturales, se necesita que tanto el Estado como los ciudadanos opten por mecanismos que sean legales, justos pero también oportunos, agiles y eficientes ante una posible afectación. El acta de conciliación extrajudicial, vinculante para las partes, podría contener, de ser el caso, la obligación de que el que afecta, restaure la situación a su estado anterior y se haga cargo del resarcimiento por el daño causado, sea este por acción u omisión.

Es necesario restaurar la confianza de la población en el Estado y los inversionistas en la atención y solución de los conflictos sociales, esta confianza se ha visto afectado por el reiterado incumplimiento de la normatividad y de los compromisos asumidos, tanto por el Estado como las empresas interesadas en las inversiones de naturaleza extractiva, en la solución de los reclamos de las poblaciones y las demandas socio ambientales. En este contexto, desde el Derecho surge una herramienta jurídica capaz de dotar de predictibilidad y cumplimiento a los acuerdos y soluciones en materia ambiental que versen sobre derechos disponibles y cuantificables económicamente mediante el uso de la conciliación extrajudicial como mecanismo de cultura de paz declarado normativamente de interés nacional. Sus atributos de valor de sentencia de última instancia y título de ejecución establecen las garantías para el debido cumplimiento de los compromisos asumidos por las partes, logrando así que la explotación de los recursos naturales y demás actividades productivas se realicen en un contexto de paz social, tranquilidad pública y respeto de los derechos.

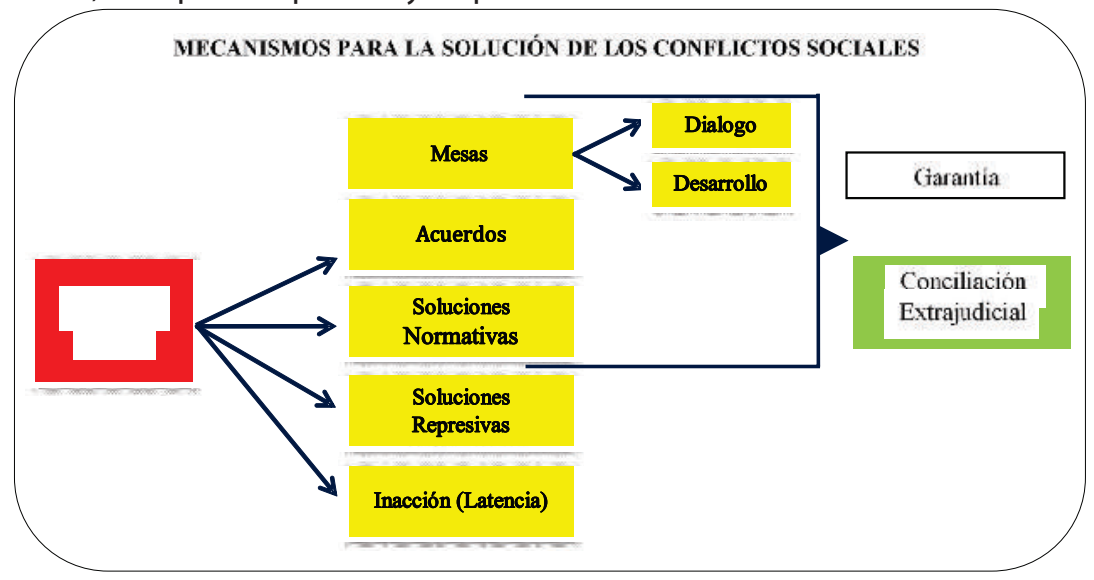

Fuente: Elaboración propia 


\section{Conclusiones}

a. Históricamente, en el Perú, se han registrado y existen diversos conflictos sociales, especialmente de naturaleza socioambiental vinculados a la actividad minera, muchos de ellos pendientes de solución; los reclamantes permanentemente denuncian la falta de cumplimiento de los acuerdos arribados, así como, dificultades para exigir el cumplimiento de los mismos en la etapa postconflicto. Esta situación genera que las controversias entren en un estado de latencia constituyendo una amenaza continua para las actividades económicas, principalmente extractivas, que motivaron el conflicto.

b. Frente a la permanencia de los conflictos sociales, sus secuelas de violencia y politización, es necesario implementar medidas que puedan brindar soluciones duraderas en la atención de las reivindicaciones o demandas de las comunidades reclamantes. Ello será posible si se garantiza de alguna forma el cumplimiento de los acuerdos arribados por las partes, luego de prolongadas y en muchos casos difíciles negociaciones; es en este momento que surge la conciliación extrajudicial como método autocompositivo de conflictos donde prima la autonomía de la voluntad de las partes y cuyos resultados plasmados en el acta de conciliación adquieren valor legal, mérito de sentencia de última instancia y título de ejecución para demandar el cumplimiento coercitivo de los compromisos asumidos con intervención de la autoridad judicial.

c. El Derecho ofrece una herramienta jurídica capaz de dotar de predictibilidad y cumplimiento a los acuerdos y soluciones en materia de conflictos sociales, que versen sobre derechos disponibles y cuantificables económicamente, mediante el uso de la Conciliación Extrajudicial como mecanismo de cultura de paz declarado normativamente de interés nacional.

d. La Conciliación Extrajudicial es un mecanismo de seguridad jurídica que viabiliza el cumplimiento de los acuerdos, siempre y cuando estos versen sobre derechos disponibles, restaurando, de esta manera, la confianza de la población en el Estado y asegurando un clima de tranquilidad para el desarrollo de las inversiones.

e. La Conciliación Extrajudicial es una herramienta dotada de virtudes legales para identificar los problemas sociales y prevenir que los conflictos converjan en actos de violencia, al posibilitar soluciones directas y proporcionales a las necesidades y demandas de las partes.

f. Optar por la Conciliación Extrajudicial es promover la cultura de paz mediante el uso del dialogo como presupuesto para llegar a un acuerdo consensual, que será plasmado en el Acta de Conciliación correspondiente y será "ley entre las partes", debido a su carácter de ejecutable judicialmente.

g. En materia de conflictos sociales existe un largo camino por recorrer, siendo 
un aspecto esencial para su solución el compromiso del Estado, las empresas inversionistas y la población con la búsqueda de una cultura de paz, que trascienda a la solución de los conflictos sociales y considere temas medio ambientales que involucran derechos colectivos; en este contexto, surge, como una garantía de solución y cumplimiento, el Acta de Conciliación Extrajudicial, más aun teniéndose en cuenta que los conflictos sociales se incrementaran debido a la escasez de los recursos naturales, el crecimiento de la población, las demandas sociales, los altos índices de contaminación, entre otros factores.

\subsection{Recomendaciones}

a. A las partes de los conflictos sociales: Se requiere que comunidades, empresas y Estado; internalicen la necesidad de una cultura de paz que garantice las condiciones para el progreso de las actividades económicas en un clima ambientalmente responsable, orientado al desarrollo económico y social del país.

b. Al órgano legislativo: Es necesario introducir modificaciones legales dirigidas a mejorar el marco normativo de la Conciliación Extrajudicial en el Perú, como un mecanismo alternativo de solución de conflictos, aplicable al complejo mundo de los conflictos sociales, se requiere flexibilizar el ritualismo y formalidades de los mecanismos conciliatorios. Así por ejemplo, en los conflictos mineros donde se enfrentan comunidades, empresas y Estado, y cuyo epicentro se encuentra por lo general en las zonas rurales lejos de los centros urbanos donde no existen Centros de Conciliación, habría que dotar de algún tipo de flexibilidad normativa para permitir que las audiencias (entre otros detalles) se realicen fuera del Centro de Conciliación ya que los formalismos actualmente existentes impedirían su aplicación a la mayor parte de casos.

c. Al órgano rector del Sistema Conciliatorio Nacional: El Ministerio de Justicia y Derechos Humanos ejerce la rectoría del Sistema Conciliatorio Nacional, como instancia normativa reglamentaria, administrativa y disciplinaria; funciones que desarrolla a través de la Dirección General de Defensa Publica y la Dirección de Mecanismos Alternativos de Solución de Conflictos, las mismas que actúan como segunda y primera instancia respectivamente. Estas instancias administrativas y de gestión del Sistema Conciliatorio deberán de adecuar sus facultades de control a las condiciones especiales que ofrecen los conflictos sociales dado el interés público existente en alcanzar la solución de los mismos y la necesidad de promocionar su uso y aplicación en aras de la seguridad jurídica y consolidación de la paz social mediante el cumplimiento efectivo de los acuerdos.

d. A los Centros de Conciliación: Los operadores jurídicos del Sistema Conciliatorio Nacional deberán identificarse con el compromiso de participar 
en los asuntos de su competencia para resolver los conflictos sociales de sus regiones o ámbitos territoriales, ofreciendo y perfeccionando sus capacidades conciliatorias para alcanzar la solución a los mismos y convirtiéndose en garantes del cumplimiento de los acuerdos mediante la institución de la Conciliación Extrajudicial y los poderes coercitivos que conlleva la ejecución de sus actas. El conciliador debe salir a la búsqueda del conflicto social y ofrecer su conocimiento y posicionamiento legal para intervenir en la solución de las controversias, en el marco de sus competencias y en materias que versen sobre derechos disponibles.

\section{Bibliografía}

ATIENZA RODRIGUEZ, M. (12 de Noviembre de 2013). "Una oportunidad perdida". "Juridica" - El Peruano, 14.

AZABACHE PEÑA, J. (24 de Abril de 2018). "La importancia de los centros de conciliación". "Jurídica" - El Peruano, 8.

BETANCOUR RODRIGUEZ, A. (2002). "Instituciones de Derecho Ambiental - Material para el curso de Derecho Ambiental". Costa Rica.

Biological Psychiatry. (31 de Enero de 2018). Obtenido de "Exposición a la contaminación del aire durante la vida fetal, la morfología cerebral y la función cognitiva en niños en edad escolar": https://www.sciencedirect.com/science/ article/pii/S0006322318300647

CHANG, R. (08 de Abril de 2016). Foro Económico. Obtenido de "Los costos económicos de los conflictos mineros en el Perú": http://focoeconomico. org/2016/04/08/los-costos-economicos-de-los-conflictos-mineros-en-el-peru/

COMERCIO, O. M. (2010). Informe sobre el Comercio Mundial 2010. Obtenido de B. Recursos naturales: definiciones, estructura del comercio y globalización: https:// www.wto.org/spanish/res_s/booksp_s/anrep_s/wtr10-2b_s.pdf

CONGRESO DE LA REPÚBLICA. (s.f.). Ley de Conciliación Extrajudicial - Ley №26872. Obtenido de OSCE: http://www.osce.gob.pe/htmls/conciliacion/ leyconciliacionextraj.htm

DE ECHAVE C., J. (22 de Enero de 2018). "¿Qué está pasando en Las Bambas?". Observatorio de Conflictos Minero en el Perú. 
DEFENSORÍA DEL PUEBLO. (24 de Noviembre de 2015). Blog de la Defensoría del pueblo. Obtenido de http://www.defensoria.gob.pe/blog/que-es-un-conflictosocial/

DEFENSORÍA DEL PUEBLO. (2017). 21ํㅡㄹ Observatorio de Conflictos Mineros en el Perú. Obtenido de Reporte Segundo Semestre 2017: http://www.grufides. org/sites/default/files//documentos/reportes_semestrales/Revista-Informe-deConflictos-Mineros-VI-2.pdf

DEFENSORÍA DEL PUEBLO. (s.f.). Defensoría del Pueblo. Obtenido de "Conflictos Sociales": https://www.defensoria.gob.pe/temas.php?des=3

DIARIO EL ESPECTADOR. (18 de Noviembre de 2017). El Espectador. Obtenido de "Una de cada cuatro muertes en el mundo se debe a la contaminación": https:// www.elespectador.com/noticias/medio-ambiente/una-de-cada-cuatro-muertesen-el-mundo-se-debe-la-contaminacion-articulo-723884

GONZALES BALLAR, R. (2001). "Temas de Derecho Ambietal". San José de Costa Rica: Editorial Investigaciones Jurídicas S.A.

HILMAN, K. -H. (2001). "Diccionario Enciclopédico de Sociología". Barcelona: Herder.

INSTITUTO PERUANO DE ECONOMÍA. (Julio de 2015). "El Costo Económico de la no ejecución de los proyectos mineros por conflictos sociales y/o trabas burocráticas". Lima.

LEWIS, C. (1961). "Las funciones del conflicto social". México: Fondo de Cultura Económica.

Ley N²861 1. (s.f.). Obtenido de Ley General del Ambiente: http://www.ana.gob.pe/ sites/default/files/normatividad/files/ley_n-28611.pdf

LEY No 28322. (s.f.). Obtenido de http://www2.congreso.gob.pe/sicr/cendocbib/ con3_uibd.nsf/93D7D59B1B826A0E052578C5005F172F/\$FILE/Ley_28322.pdf

MEDINA ROSPIGLIOSI, R. G. (s.f.). Mediate. Obtenido de "Primer decenio de la conciliacion extrajudicial en el Peru, problemas y propuestas de cambio": https:// www.mediate.com/articles/conciliacion_extrajudicial.cfm\#bio

PESQUEIRA LEAL, J. (16 de Setiembre de 2015). "El Estado debe incentivar la comunicación entre empresa y comunidad para evitar malos entendidos". Revista 
Rumbo Minero. Obtenido de http://www.rumbominero.com/noticias/mineria/ el-estado-debe-incentivar-la-comunicacion-entre-empresa-y-comunidad-paraevitar-malos-entendidos/

PODER EJECUTIVO DEL PERU. (01 de Septiembre de 2008). Decreto Legilsativo № 1071. Obtenido de Decreto Legislativo que norma el arbitraje: http://portal.osce. gob.pe/arbitraje/sites/default/files/Documentos/Legislacion_aplicable/DL-1071ley-que-norma-el-arbitraje.pdf

PRATT FAIRCHIL, H. (1974). "Diccionario de Sociología". México: Fondo de Cultura Económica.

PUMA ALMANZA, L. (s.f.). "Mesas de Diálogo y Mesas de Desarrollo: espacios para el ejercicio democrático". Obtenido de ProDiálogo - Prevención y Resolución de Conflictos: http://prodialogo.org.pe/blog/mesas-de-di\%C3\%A1 logo-y-mesas-dedesarrollo-espacios-para-el-ejercicio-democr\%C3\%A1 tico

REAL ACADEMIA ESPAÑOLA. (s.f.). Real Academia Española. Recuperado el Mayo de 2018, de Diccionario de la Lengua Española: http://dle.rae.es/?id=DxZ9aNj

ROSA H., H. D. (1999). "Valoración y pago por servicios ambientales: Las experiencias de Costa Rica y El Salvador". Obtenido de http://www.prisma.org.sv/pubs/prisma35. pdf

VELASQUEZ MUÑOZ, C. J. (2004). "Conciliación de Conflictos Ambientales". Obtenido de Revista de Derecho - Universidad del Norte: https://dialnet.unirioja. es/descarga/articulo/2347489.pdf 